\title{
DIFFERENTIABILITY ALMOST EVERYWHERE OF FUNCTIONS OF SEVERAL VARIABLES ${ }^{1}$
}

\author{
G. V. WELLAND ${ }^{2}$
}

1. The purpose of this paper is to extend the results of C. J. Neugebauer in [3], where necessary and sufficient conditions for a measurable function of a real variable to be equivalent to one which is differentiable are given, to the case of higher order differentiation and to functions of several real variables.

Let $f: E_{n} \rightarrow R$ be a measurable function from $n$-dimensional Euclidean space to the reals. $Q$ will denote a cube in $E_{n}$; and if $E$ is a measurable set in $E_{n}$, we denote its measure by $|E|$. All functions and sets will be assumed to be measurable. Suppose that we can write $f\left(x_{0}+t\right)$ $=P_{x_{0}}(t)+R_{x_{0}}(t)$ where $P_{x_{0}}(t)$ is a polynomial of degree $\leqq k-1$, then we say $f$ has a $k-1$ derivative at $x_{0}$ in the $L^{p}$ sense $(1 \leqq p \leqq \infty)$ if

$$
\left\{\frac{1}{\rho^{n}} \int_{|t| \leq \rho}\left|R_{x_{0}}(t)\right|^{p} d t\right\}^{1 / p}=o\left(\rho^{k-1}\right), \quad \rho \rightarrow 0 .
$$

We write

$$
\Delta_{x_{0}}^{k}(f, t)=\Delta_{x_{0}}^{k}(t)=2 \stackrel{k}{k}(t)|t|^{k}=R_{x_{0}}(t)+(-1)^{k-1} R_{x_{0}}(-t) .
$$

If $R_{x_{0}}(t)=o\left(|t|^{k-1}\right)$ as $|t| \rightarrow 0$, we say $f$ has a $k-1$ derivative at $x_{0}$; and if $R_{x_{0}}(t)=o\left(|t|^{k-1}\right)$ as $|t| \rightarrow 0$ through a set of Lebesgue density 1 at $x_{0}$, we say $f$ has an approximate $k-1$ derivative at $x_{0}$. Finally, if $R_{x_{0}}(t)=o\left(|t|^{k-1}\right)$ for each $x_{0}$ in a set $E$ provided $t \rightarrow 0$ through a set whose complement has measure zero, we say $f$ has a $(k-1)_{*}$ derivative for each $x_{0}$ in $E$.

With these remarks we can state the following known results.

TheOREM A (SEE [6]). $f$ has a kth derivative in the $L^{2}$ sense for almost every $x_{0}$ in $E,|E|>0$, if and only if for almost every $x_{0}$ in $E$ there exists an $\eta_{x_{0}}=\eta>0$ such that

$$
\int_{|t| \leqq \eta} \frac{\left|\Delta_{x_{0}}^{k}(t)\right|^{2}}{|t|^{n+2 k}} d t<\infty .
$$

TheOREM B. $f$ has a kth derivative in the $L^{p}$ sense $(2 \leqq p<\infty)$ at almost every $x_{0}$ in $E,|E|>0$, if and only if for almost every $x_{0}$ in $E$ there exists $\eta_{x_{0}}=\eta>0$ such that

Received by the editors November 30, 1966.

1 This is a portion of the author's doctoral dissertation at Purdue University.

2 Partially supported by NSF Grant GP-1665. 


$$
\int_{|t| \leqq \eta} \frac{\left|\Delta_{x_{0}}^{k}(t)\right|^{2}}{|t|^{n+2 k}} d t<\infty, \quad \int_{|t| \leqq \eta} \frac{\left|\Delta_{x_{0}}^{k}(t)\right|^{p}}{|t|^{n+p k}} d t<\infty .
$$

Theorem $A$ is due to Stein and Zygmund and was originally proved in [5] for the case of a function of a single variable. In [6] it is shown how it can be extended as indicated here. Theorem B was proved in [7] for the case $k=1$. With Theorem A one can prove Theorem B by using a similar argument to that found in [5, p. 280].

The theorem we wish to prove is the following one.

THEOREM 1. $f$ is equivalent to a function which has a kth derivative almost everywhere in a set $E,|E|>0$, if and only if for almost every $x$ in $E$ there is an $\eta_{x}=\eta>0$ such that the sequence

$$
\left\{\int_{|t| \leq \eta} \frac{\left|\Delta_{x_{0}}^{k}(t)\right|^{p}}{|t|^{n+p k}} d t\right\}^{1 / p}, \quad p=2,3, \cdots
$$

is bounded.

2. We need some definitions and lemmas to enable us to prove these theorems. Let $E_{y}=\{x: f(x)>y\}$ for real values of $y$. Set $g\left(x_{0}\right)$ $=\inf _{y}\left\{y:\left|E_{y} \cap Q\right|=o(|Q|)\right.$ as $\left.|Q| \rightarrow 0, x_{0} \in Q\right\}$. Then $g: E_{n} \rightarrow R$ is a function, is called the upper boundary of $f$ (see [1]), and satisfies the properties:

( $\left.\mathrm{P}_{1}\right) f(x)=g(x)$ at every point of approximate continuity of $f$, and hence $f$ is equivalent to $g$;

$\left(\mathrm{P}_{2}\right)$ for $x_{0}$ in $E_{n}$ and $\epsilon>0, \eta>0$, the set $\left\{x:\left|x-x_{0}\right|<\eta\right.$ and $\left.\left|g(x)-g\left(x_{0}\right)\right|<\epsilon\right\}$ has positive measure.

Lemma 1. Let $E$ be a measurable set and suppose f has $a(k)_{*}$ derivative at each point of $E$. Then $f$ is equivalent to a function which has a $k$ th derivative at each point of $E$.

Proof. Let $g$ be the upper boundary of $f$. Then $f$ is equivalent to $g$ and $f(x)=g(x)$ at every point of $E$. Let $x_{0}$ be in $E$ and let $\epsilon>0$ be given. We may assume $P_{x_{0}}(t)=0$. There is a $\delta>0$ and a set $N$, $|N|=0$, and $N \subset\left\{x:\left|x-x_{0}\right|<\delta\right\}$ such that $\left|g\left(x_{0}+t\right)\right| \leqq \epsilon|t|^{k}$ provided $x_{0}+t \notin N$ and $|t|<\delta$. If $N \neq \varnothing$, there is a $\sigma>0$ and a point $t_{0}$, $\left|t_{0}\right|<\delta$, and $\left(x_{0}+t\right) \in N$ such that $\left|g\left(x_{0}+t_{0}\right)\right| \geqq(\epsilon+\sigma)\left|t_{0}\right|^{k}$. By property $\left(\mathrm{P}_{2}\right)$ there is a sequence $\left\{t_{n}\right\}, t_{n} \rightarrow t_{0}$ and $x_{0}+t_{n}$ is not in $N$, such that $g\left(x_{0}+t_{n}\right) \rightarrow g\left(x_{0}+t_{0}\right)$. This implies $\left|g\left(x_{0}+t_{0}\right)\right| \leqq \epsilon\left|t_{0}\right|{ }^{k}$ from which we conclude $N=\varnothing$, and the lemma follows.

Lemma 2. Assume that for $x \in E,|E|>0$, there is a set $N_{x},\left|N_{x}\right|=0$, such that $\Delta_{x}^{\boldsymbol{k}}(t)=O\left(|t|^{k}\right)$ as $|t| \rightarrow 0$ for $t$ in $N_{x}$. Then there exists a set $N$, 
$|N|=0$, such that $\Delta_{x}^{\mathbf{k}}(t)=O\left(|t|^{k}\right)$ as $|t| \rightarrow 0$ with $(x \mp t) \notin N$ for each $x$ in $E$.

Proof. Let $N$ be the set of points at which $f$ is not approximately continuous. $|N|=0$, and it can easily be shown that $\Delta_{x}^{\boldsymbol{k}}(t)=O\left(|t|^{k}\right)$ as $|t| \rightarrow 0$ with $(x \mp t) \notin N$ for each $x$ in $E$.

Lemma 3. Let $f: E_{n} \rightarrow R$ be measurable and $1 \leqq p \leqq \infty$. Then at all points $x$, at which $f$ has a kth derivative in the $L^{p}$ sense, $f$ has an approximate kth derivative.

LEMMA 4. Let $f: E_{n} \rightarrow R$ be measurable such that at each $x_{0}$ in a set $E$, $|E|>0, f$ has a kth approximate derivative and that the polynomial $P_{x_{0}}(t)$ is zero. Suppose that for each $x_{0}$ in $E$ there is a set $N_{x_{0}}$ such that $\left|N_{x_{0}}\right|=0$ and $\Delta_{x_{0}}^{\mathbf{k}}(t)=O\left(|t|^{k}\right)$ as $|t| \rightarrow 0, t \notin N_{x_{0}}$. Then $f$ has $a(k)_{*}$ derivative at almost all $x_{0} \in E$.

Proof. As in Lemma 2, there is a set $N,|N|=0$, such that $\Delta_{x}^{\mathbf{k}}(t)$ $=O\left(|t|^{k}\right)$ as $|t| \rightarrow 0$ with $x \pm t \notin N$ for each $x \in E$. Let $F_{1} \subset F_{2} \subset \cdots$ be a sequence of closed sets such that $f$ restricted to $F_{i}$ is continuous, $F_{i} \subset E_{n}-N$, and $H=\bigcup_{i=1}^{\infty} F_{i}$ has full measure in every cube. Let

$E(m, j)=\left\{x \in E \cap H:\left|\Delta_{x}^{k}(t)\right|<m|t|^{k}, 0<|t|<1 / j, x+t \in H\right\}$.

One can show these sets are measurable (see e.g. [4]). We will show that at each point of density of $E(m, j) f$ has a $(k)_{*}$ derivative, and this will complete the proof.

Assume $x_{0}$ is a point of density of $E(m, j)$. Let $\sigma>0$ be given. Then there is a set $E_{1} \subset E(m, j)$ with $x_{0}$ as a point of density, and if $x_{0}+t$ is in $E_{1}$ we have $\left|f\left(x_{0}+t\right)\right| \leqq \sigma|t|^{k}$. Let

$$
\begin{array}{r}
A_{t}=\left\{x:\left(x_{0}+x+t\right) / 2 \in E_{1}, 0<\left|x-x_{0}\right|<|t|\right. \\
\text { and } \left.\left|x_{0}+t-x\right|<(\sigma / m)^{1 / k}|t|\right\} .
\end{array}
$$

Let $\chi$ be the characteristic function of $E_{1}$, then $A_{t}$ has measure equal to

$$
\int_{\left|x_{0}+\ell-x\right|<(\sigma / m)^{1 / k}|t|} \chi\left(\frac{x_{0}+x+t}{2}\right) d x .
$$

Since $x_{0}$ is a point of density of the set $E_{1}$, this integral is asymptotically equal to $C_{n}(\sigma / m)^{n / k}|t|^{n}$ as $|t| \rightarrow 0$ where $C_{n}$ is a constant depending only on $n$. Hence there is $0<\delta<1 / j$ such that $A_{t} \cap E \neq \varnothing$ whenever $0<|t|<\delta$. Thus for every $|t|, 0<|t|<\delta$, there is an $x \in A_{t} \cap E_{1}$. Since $x \in E_{1}$ we have $|f(x)| \leqq \sigma\left|x-x_{0}\right|^{k} \leqq \sigma|t|^{k}$ and if $x_{0}+t$ is in $H$ we obtain 


$$
\begin{aligned}
\left|f\left(x_{0}+t\right)\right| & \leqq\left|f\left(x_{0}+t\right)+(-1)^{k-1} f(x)\right|+|f(x)| \\
& \leqq m\left|\left(x_{0}+t-x\right) / 2\right|^{k}+\sigma|t|^{k} \\
& \leqq m(\sigma / m)|t|^{k}+\sigma|t|^{k}=2 \sigma|t|^{k} .
\end{aligned}
$$

Hence $f$ has a $k$ th derivative at $x_{0}$ relative to the set $H$.

LeMma 5 (SEe [2, p. 18]). Let $f$ be a real valued function in $L^{p}\left(E_{n}\right)$ $(1 \leqq p \leqq \infty)$ and suppose $f$ has a kth derivative in the $L^{p}$ sense at each point of a set $E$ of positive measure. Then, for $\epsilon>0$, there is a closed set $S$, $|E-S|<\epsilon$, and two functions $f_{1}$ and $f_{2}$ such that $f=f_{1}+f_{2}$ with $f_{1} \in C^{k}$, and for $x_{0}$ in $S$,

$$
\int_{|t| \leq \rho}\left|f_{2}\left(x_{0}+t\right)\right|^{p} d t=o\left(\rho^{p k+n}\right), \quad \rho \rightarrow 0 .
$$

3. We are now ready to give the proof of Theorem 1. First let us assume that there is a function $g$ equivalent to $f$ which has a $k$ th derivative almost everywhere in $E$. By Theorem $\mathrm{A}$, for almost every $x$ in $E$, there is an $\eta_{x}=\eta>0$ such that

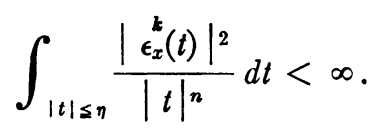

We will show that the sequence (4) is bounded at every point $x$ for which $f(x)=g(x), g$ has a $k$ th derivative and (5) holds. Since $g$ has a $k$ th derivative, $\Delta_{x}^{k}(g, t)=o\left(|t|^{k}\right)$. Also, since $f(x)=g(x)$ almost everywhere, there is an $\eta^{\prime}, 0<\eta^{\prime}<\eta$, and a set $N_{x}$ of zero measure such that $\Delta_{x}^{\mathbf{k}}(t)<|t|^{k}$ for $|t|<\eta^{\prime}$ and $t \notin N_{x}$. Thus for $p \geqq 2$

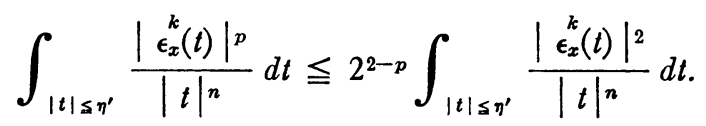

Hence, (4) is bounded with $\eta$ replaced by $\eta^{\prime}$.

Now assume (4) is bounded, with $M<\infty$ as bound. Then we have ess $\sup \left|\Delta_{x_{0}}^{k}(t)\right| \leqq M|t|^{k}, 0<|t|<\eta$. The finiteness of the first term of the sequence $(p=2)$ implies the existence of the $k$ th derivative in the $L^{2}$ sense almost everywhere in $E$ and that $f$ is in $L^{2}$ of a neighborhood of almost every point of $E$. Let $\epsilon>0$ be given. By Lemma 5, there is a set $S$ such that $f=f_{1}+f_{2},|E-S|<\epsilon, f_{1}$ is $C^{k}$, and for $x$ in $S$,

$$
\int_{|t| \leqq t}\left|f_{2}(x+t)\right|^{2} d t=o\left({ }_{\rho}^{2 k+n}\right) \text {. }
$$

Since $\epsilon$ is arbitrary, it is enough to show that $f_{2}$ is equivalent to a 
function which has $k$ derivatives at almost every point of $S$. Equation (6) and Lemma 3 imply that $f_{2}$ has a $k$ th approximate derivative at the points $x_{0}$ in $S$ and that the polynomial $P_{x_{0}}(t)$ for $f_{2}$ is zero. Since $f_{1}$ is $C^{k}$, it follows that $\Delta_{x}^{\mathbf{k}}\left(f_{1}, t\right)=o\left(|t|^{k}\right)$ and hence $\Delta_{x}^{\mathbf{k}}\left(f_{2}, t\right)=O\left(|t|^{k}\right)$ provided $|t|<\eta$ and $t \notin N_{x},\left|N_{x}\right|=0$. Now apply Lemma 4 and Lemma 1.

We point out that the boundedness of (4) and Theorem B imply that $f$ has a $k$ th derivative in the $L^{p}$ sense $(2 \leqq p<\infty)$ almost everywhere in $E$. The boundedness of (4) may be regarded as a condition for the existence of the $k$ th derivative in the $L^{p}$ sense $(2 \leqq p<\infty)$ almost everywhere in $E$ and uniformly in $p$. This is precisely what is needed to pass to the $p=\infty$ case, as was pointed out in [3] for a function of a single variable.

\section{BIBLIOGRAPHY}

1. H. Blumberg, The measurable boundaries of an arbitrary function, Acta Math. 65 (1935), 263-282.

2. A. P. Calderón and A. Zygmund, Local properties of solutions of elliptic partial differential equations, Studia Math. 20 (1961), 171-225.

3. C. J. Neugebauer, Differentiability almost everywhere, Proc. Amer. Math. Soc. 16 (1965), 1205-1210.

4. - Symmetric and smooth functions of several variables, Math. Ann. 153 (1964), 285-292.

5. E. M. Stein and A. Zygmund, On the differentiability of functions, Studia Math. 23 (1964), 247-283.

6. E. M. Stein, Singular integrals, harmonic functions, and differentiability properties of functions of several vaiiables, Symposium on Singular Integrals, Chicago, Ill., 1966.

7. R. L. Wheeden, On the n-dimensional integral of Marcinkiewicz, J. Math. Mech. 14 (1965), 61-70.

De PAUL Lniversity 